\title{
ON THE MULTIPLICATION OF TENSOR FIELDS
}

W. GREUB AND E. STAMM ${ }^{1}$

Let $M$ be a paracompact $n$-dimensional manifold of class $C^{k+1}$, $T(x)$ the tangent space of the point $x \in M$ and $T(x)^{*}$ the dual space. A real valued $(p+r)$-linear function $\Phi(x)$ with $p$ arguments in $T(x)$ and $r$ arguments in $T(x)^{*}$ is called a tensor of type $(p, r)$ at the point $x$. The tensors of type $(p, r)$ at $x$ form a linear space $T_{p}^{r}(x)$. The products of two tensors $\Phi \in T_{p}^{r}(x)$ and $\Psi \in T_{q}^{s}(x)$ is defined by

$$
\begin{aligned}
(\Phi \Psi)(x ; & \left.\xi_{1} \cdots \xi_{p+q}, \xi^{*_{1}} \cdots \xi^{*+s}\right) \\
= & \Phi\left(x ; \xi_{1} \cdots \xi_{p}, \xi^{* 1} \cdots \xi^{* r}\right) \\
& \cdot \Psi\left(x ; \xi_{p+1} \cdots \xi_{p+q}, \xi^{*++1} \cdots \xi^{*++\bullet}\right), \\
& \xi_{\nu} \in T(x), \xi^{*_{\mu}} \in T(x)^{*} .
\end{aligned}
$$

A tensor field of type $(p, r)$ and class $C^{k}$ on $M$ is an assignment of tensors of type $(p, r)$ to the points of $M$ such that the components with respect to a local coordinate system are $C^{k}$-functions. The set of all tensor fields of type $(p, r)$ and class $C^{k}$ is a module $T_{p}^{r}$ over the ring $F$ of $C^{k}$-functions on $M$. The multiplication of tensors induces a multiplication of tensor fields in an obvious way. Now consider the $F$-bilinear mapping

$$
T_{p}^{r} \times T_{q}^{s} \rightarrow T_{p+q}^{r+s}
$$

which is defined by the multiplication. This bilinear mapping induces a $F$-linear mapping

$$
h: T_{p}^{r} \otimes T_{q}^{8} \rightarrow T_{p+q}^{r+s}
$$

such that

$$
h(\Phi \otimes \Psi)=\Phi \cdot \Psi
$$

We shall prove in this paper the following

TheOREM. $h: T_{p}^{r} \otimes T_{q}^{s} \rightarrow T_{p+q}^{r+s}$ is an isomorphism.

For the sake of simplicity we restrict ourselves to covariant tensor

Received by the editors October 4, 1965.

1 The second author holds a fellowship of the National Research Council of Canada. 
fields, i.e. tensor fields of type $(p, 0)$ and write $T_{p}$ instead of $T_{p}^{o}$. However, the argument can be carried over word by word to the general case.

Before giving the proof of the theorem we state some corollaries and show how they can be deducted from the theorem. We are indebted to the referee for the suggestion to include the Corollaries 3 and 4.

Corollary 1. Let $\left(T_{p}^{r}\right) *$ be the dual of the F-module $T_{r}^{p}$. Then

$$
\left(T_{p}^{r}\right)^{*} \otimes\left(T_{q}^{s}\right)^{*} \cong\left(T_{p}^{r} \otimes T_{q}^{s}\right)^{*}
$$

Proof. There exists a canonical $F$-isomorphism

$$
\phi_{p}^{r}:\left(T_{p}^{r}\right)^{*} \rightarrow T_{r}^{p}
$$

(cf. Helgason, Differential geometry and symmetric spaces, Academic Press, New York, 1958; p. 15). Since all maps in the diagram

$$
\begin{array}{r}
\left(T_{p}^{r}\right)^{*} \otimes\left(T_{q}^{s}\right)^{*} \stackrel{\dot{\phi}_{p}^{r} \otimes \dot{\phi}_{q}^{*}}{\longrightarrow} T_{r}^{p} \otimes T_{*}^{q} \stackrel{h}{\longrightarrow} T_{r+\varepsilon}^{p+q} \\
\downarrow i \\
\left(T_{p}^{r} \otimes T_{q}^{*}\right)^{*} \stackrel{h^{*}}{\longrightarrow}\left(T_{p+q}^{r+\varepsilon}\right)^{*} \stackrel{\phi_{p+q}^{r+q}}{\longrightarrow} T_{r+\varepsilon}^{p+q}
\end{array}
$$

are isomorphisms it follows that

$$
\left(h^{*}\right)^{-1} \circ\left(\phi_{p+q}^{r+s}\right)^{-1} \circ h \circ\left(\phi_{p}^{r} \otimes \phi_{q}^{s}\right)
$$

is an isomorphism of $\left(T_{p}^{r}\right)^{*} \otimes\left(T_{q}^{s}\right) *$ onto $\left(T_{p}^{r} \otimes T_{q}^{s}\right)^{*}$.

CoRollary 2. The abstract pth tensorial power $\otimes^{p} T_{1}$ is isomorphic to $T_{p}$ under $h$.

Proof. This follows immediately from the theorem.

Symmetric Tensors. Let $S: \otimes^{p} T_{1} \rightarrow \otimes^{p} T_{1}$ be the operator of symmetry defined by

$$
S\left(\omega^{1} \otimes \cdots \otimes \omega^{p}\right)=\frac{1}{p !} \sum_{\sigma} \omega^{\sigma(1)} \otimes \cdots \otimes \omega^{\sigma(p)}
$$

where $\sigma$ runs through all permutations of $p$ objects. The symmetric product $\bigvee^{p} T_{1}$ is defined to be the $F$-module $\operatorname{Im} S \subset \otimes^{p} T_{1}$. Denote by $i: \bigvee^{p} T_{1} \rightarrow \otimes^{p} T_{1}$ the inclusion homomorphism. On the other hand consider the submodule $S_{p} \subset T_{p}$ of symmetric tensors; let $i^{\prime}: S_{p} \rightarrow T_{p}$ 
be the inclusion homomorphism. $\Phi$ is in $S_{p}$ if and only if for any permutation $\sigma$ and any vector fields $\xi_{i}, \cdots, \xi_{p}$ we have $(\sigma \Phi)\left(\xi_{1}, \cdots, \xi_{p}\right)$ $=\Phi\left(\xi_{\sigma(1)}, \cdots, \xi_{\sigma(p)}\right)=\Phi\left(\xi_{1}, \cdots, \xi_{p}\right)$.

Corollary 3. The F-isomorphism $h: \otimes^{p} T_{1} \rightarrow T_{p}$ induces an $F$ isomorphism $\bar{h}: \bigvee^{p} T_{1} \rightarrow S_{p}$ such that $h \circ i=i^{\prime} \circ \bar{h}$.

Proof. One easily verifies that $\operatorname{Im}(h \circ i)=\operatorname{Im} i^{\prime}$; then defines $\bar{h}=i^{\prime-1} \circ h \circ i$. q.e.d.

Exterior Forms. The $p$ th exterior power $\Lambda^{p} T_{1}$ is the quotient $F$ module $\otimes^{p} T_{1} / N$ where $N \subset \otimes^{p} T_{1}$ is the submodule generated by the elements $\Phi \in \otimes^{p} T_{1}$ such that $\tau \Phi=\Phi$ for some transposition $\tau$. The operator $Q$ of antisymmetrization is defined by

$$
\propto \Phi=\frac{1}{p !} \sum_{\sigma} \epsilon_{\sigma} \cdot \sigma \Phi
$$

where $\sigma$ runs over all permutations of $p$ objects and $\epsilon_{\sigma}$ is the sign of the permutation $\sigma$. Let $N_{1} \subset \otimes^{p} T_{1}$ be the kernel of $a$. According to Bourbaki, Algèbre, Chapter III, p. 60, $2^{e}$ edition, we have $N \subset N_{1}$. But on the other hand by Proposition 3 in Bourbaki, loc. cit., p. 58, for any $\Phi \in \otimes^{p} T_{1}$ and any permutation $\sigma$, we have $\Phi-\epsilon_{\sigma} \cdot \sigma \Phi \in N$. Therefore

$$
\sum_{\sigma}\left(\Phi-\epsilon_{\sigma} \cdot \sigma \Phi\right) \in N
$$

or $p ! \Phi-a \Phi \in N$. Hence, if $\Phi \in N_{1}$, then $\Phi \in N$. Whence $N_{1}=N$.

Now let $h: \otimes^{p} T_{1} \rightarrow T_{p}$ be the isomorphism of our main theorem, $\pi: \otimes^{p} T_{1} \rightarrow \wedge^{p} T_{1}$ the canonical projection homomorphism and $Q^{\prime}: T_{p} \rightarrow A_{p} \subset T_{p}$ the antisymmetrization map in $T_{p} . A_{p}$ consists of the antisymmetric tensors or global $p$-forms.

Corollary 4. $h: \otimes^{p} T_{1} \rightarrow T_{p}$ induces an F-isomorphism $\bar{h}: \wedge^{p} T_{1}$ $\rightarrow A_{p}$ such that $\bar{h} \circ \pi=Q^{\prime} \circ h$.

Proof. We have shown that $N_{1}=N$ or equivalently ker $Q=\operatorname{ker} \pi$ $=N$. It is easy to verify by computation that $h \circ Q=a^{\prime} \circ h$. Then $k(\operatorname{ker} \pi)=h(\operatorname{ker} Q)=\operatorname{ker} Q^{\prime}$ and this proves that $\bar{h}$ exists and is an isomorphism.

We proceed to the proof of the main theorem.

Lemma I. Let $U_{\alpha}$ be a system of coordinate neighborhoods on $M$ such that

$$
U_{\alpha} \cap U_{\beta}=\varnothing \quad \text { if } \alpha \neq \beta
$$


and $A_{\alpha}$ be a compact subset of $U_{\alpha}$. Then there exists a system of $n$ tensor fields $\omega^{i} \in T_{1}$ such that the $n$ tensors $\omega^{i}(x)$ are linearly independent for every $x \in \cup_{\alpha} A_{\alpha}$.

Proof. In each $U_{\alpha}$ there exists a system of $n$ tensor fields $\tilde{\omega}_{\alpha}^{i}$ of order 1 in $U_{\alpha}$ such that the tensors $\tilde{\omega}_{\alpha}^{i}(x),(i=1 \cdots n)$ are linearly independent at every point $x \in U_{\alpha}$. Now let $h_{\alpha}$ be a $C^{k}$-function on $M$ such that the carrier of $h_{\alpha}$ is compact and contained in $U_{\alpha}$ and that

$$
h_{\alpha}=1 \text { in } A_{\alpha} \text {. }
$$

Define $\omega_{\alpha}^{i}$ by

$$
{\stackrel{i}{\omega_{\alpha}}}^{i}=\left\{\begin{array}{cl}
h_{\alpha} \tilde{\omega}_{\alpha}^{i} & \text { in } U_{\alpha}, \\
0 & \text { in } M-U_{\alpha}
\end{array}\right.
$$

and $\omega^{i}$ by

$$
\omega^{i}=\sum_{\beta} \stackrel{i}{\omega_{\beta}}
$$

Then $\omega^{i}$ is a tensor field of order 1 on $M$. Now let $x \in \mathrm{U}_{\alpha} A_{k}$ be an arbitrary point. Since the compact sets $A_{\alpha}$ are mutually disjoint the point $x$ belongs to precisely one of them, say to $A_{\alpha}$. This implies that

$$
\omega^{i}(x)=h_{\alpha}(x) \tilde{\omega}_{\alpha}^{i}(x)=\tilde{\omega}_{\alpha}^{i}(x)
$$

i.e., the tensors $\omega^{i}(x)$ are linearly independent.

Lemma II. Consider the sets $U_{\alpha}$ and $A_{\alpha}$ given in Lemma I. Then there exists a system of $n$ tensor fields $\omega^{i} \in T_{1}$ with the following property: Every tensor field $\phi \in T_{p}$ whose carrier is contained in $\mathrm{U}_{\alpha} A_{\alpha}$ can be written in the form

$$
\phi=\sum_{(\nu)} \lambda_{\nu_{1}} \cdots \nu_{p} \omega^{\nu_{1}} \cdots \omega^{p}
$$

where the $\lambda_{\nu_{1}} \ldots \nu_{p}$ are scalar functions on $M$ whose carriers are contained in $\mathrm{U}_{\alpha} A_{\alpha}$.

Proof. Choose a system of open sets $B_{\alpha}$ with compact closure such that

$$
A_{\alpha} \subset B_{\alpha} \subset \bar{B}_{\alpha} \subset U_{\alpha} .
$$

Applying Lemma I to the compact sets $\bar{B}_{\alpha}$ we obtain $n$ tensor fields $\omega^{i} \in T_{i}$ such that the tensors $\omega^{i}(x)$ are linearly independent for every 
$x \in \mathrm{U}_{\alpha} B_{\alpha}$. Hence, we can write

$$
\phi(x)=\sum_{(\nu)} \tilde{\lambda}_{\nu_{1} \cdots \nu_{p}}(x) \omega^{\nu_{1}}(x) \cdots \omega^{\nu_{p}}(x), \quad x \in \bigcup_{\alpha} B_{\alpha},
$$

where the coefficients are $C^{k}$-functions in $U_{\alpha} B_{\alpha}$. Since the carrier of $\Phi$ is contained in $U_{\alpha} A_{\alpha}$ the same must be true for every function $\tilde{\lambda}_{\nu_{1} \cdots v_{p}}$. Hence, a system of $C^{k}$-functions $\lambda_{\nu_{1} \cdots p_{p}}$ can be defined on $M$ by

$$
\lambda_{p_{1} \cdots p_{p}}= \begin{cases}\tilde{\lambda}_{\nu_{1} \cdots p_{p}} & \text { in } \bigcup_{\alpha} B_{\alpha} \\ 0 & \text { in } M-\bigcup_{\alpha} B_{\alpha} .\end{cases}
$$

Then

$$
\Phi(x)=\sum_{(\nu)} \lambda_{\nu_{1}} \cdots \nu_{p}(x) \omega^{\nu_{1}}(x) \cdots \omega^{\nu p}(x)
$$

for every point $x \in M$. In fact, if $x \in \cup_{\alpha} B_{\alpha}$, the relation (2) follows from (1) and otherwise both sides of (2) are zero.

Lemma III. With $U_{\alpha}$ and $A_{\alpha}$ as in Lemma I consider any $2 r$ tensor fields $\Phi^{i} \in T_{p}$ and $\Psi^{i} \in T_{p}$ where the carriers of the $\Phi^{i}$ are contained in $\mathrm{U}_{\alpha} A_{\alpha}$. Then the relation

$$
\sum_{j} \Phi^{j} \cdot \Psi^{j}=0
$$

implies that

$$
\sum_{j} \Phi^{j} \otimes \Psi^{j}=0
$$

Proof. Choose the $B_{\alpha}$ as in Lemma II and let $\omega^{i}$ be the tensor fields constructed in Lemma II. Then $\Phi^{i}$ can be written as

$$
\Phi^{j}=\sum_{(\nu)} \lambda_{\nu_{1}}^{j} \cdots \nu_{p} \omega^{\nu_{1}} \cdots \omega^{\nu_{p}}
$$

It follows from (3) that

$$
\sum_{j} \sum_{(\nu)} \lambda_{\nu_{1}}^{j} \cdots \nu_{p} \omega^{\nu_{1}} \cdots \omega^{\nu_{p}} \cdot \Psi^{j}=0 .
$$

Since the tensors $\omega^{i}(x)$ are linearly independent for every $x \in \bigcup_{\alpha} B_{\alpha}$ the relation (4) implies that 


$$
\sum \lambda_{\nu_{1}}^{j} \ldots \nu_{p}(x) \Psi^{j}(x)=0, \quad x \in \bigcup_{\alpha} B_{\alpha} .
$$

by Lemma II the carrier of $\lambda_{\nu_{1}}^{j} \ldots v_{p}$ is contained in $U_{\alpha} A_{\alpha}$ and hence in $\bigcup_{\alpha} B_{\alpha}$. Thus (5) holds for every $x \in M$, i.e.

$$
\sum_{j} \lambda_{p_{1} \ldots p_{p}}^{j} \Psi^{j}=0 \text {. }
$$

Now the bilinearity of the tensor product yields

$$
\begin{aligned}
\sum_{j} \Phi^{j} \otimes \Psi^{j} & =\sum_{j}\left(\sum_{(\nu)} \lambda_{\nu_{1} \cdots \nu_{p}}^{j} \omega^{\nu_{1}} \cdots \omega^{\nu_{p}}\right) \otimes \Psi^{j} \\
& =\sum_{(\nu)}\left(\omega^{\nu_{1}} \cdots \omega^{\nu_{p}} \otimes \sum_{j} \lambda_{\nu_{1}}^{j} \cdots \nu_{p} \Psi^{j}\right)=0 .
\end{aligned}
$$

Lemma IV. Let $M$ be a paracompact $n$-dimensional manifold. Then there exists a locally finite covering by open sets $V_{\alpha}^{\mathbf{k}}$ where $k=0,1, \cdots, n_{0}$ $\left(n_{0} \leqq n\right)$ and $\alpha \in J_{k}\left(J_{k}\right.$ index sets) subject to the following conditions:

(i) $\bar{V}_{\alpha}^{k}$ is compact,

(ii) $V_{\alpha}^{\mathbf{k}}$ is contained in a coordinate neighborhood,

(iii) $V_{\alpha}^{k} \cap V_{\beta}^{k}=\varnothing$ for $\alpha \neq \beta$.

PROof. Since $M$ is a manifold, we may consider the covering $\{U\}$ consisting of all relatively compact coordinate neighborhoods. $M$ is paracompact and hence there is a locally-finite refinement $\{S\}$ of $\{U\}$. As a paracompact space, $M$ is normal; $M$ has dimension $n$, hence $\{S\}$ has a refinement $\left\{R_{\mu}\right\}$ of order $\leqq n$. (See C. H. Dowker, Amer. J. Math. (1947), p. 211, together with W. Hurewicz, Dimension theory, Princeton Univ. Press, Princeton, N. J., 1941; Theorem V8, p. 67.) Again, since $M$ is paracompact, there is a locally finite refinement $\left\{W_{\mu}\right\}$ of $\left\{R_{\mu}\right\}$ with index set a subset of the former index set and $W_{\mu} \subset R_{\mu}$. For if $\left\{Z_{\beta}\right\}$ is a locally-finite refinement of $\left\{R_{\mu}\right\}$ choose $\mu(\beta)$ such that $Z_{\beta} \subset R_{\mu(\beta)}$ and put $W_{\mu}=\bigcup_{\mu(\beta)=\mu} Z_{\beta}$. Then $\left\{W_{\mu}\right\}$ is locally finite and of order $n_{0} \leqq n$. There exists a partition of unity $\left\{\phi_{\mu}\right\}$ with carrier $\phi_{\mu} \subset W_{\mu}$. Of course carrier $\phi_{\mu}$ is compact. Given $k+1$ different indices $\mu_{0}, \cdots, \mu_{k}$ put $\alpha=\left(\mu_{0} \cdots \mu_{k}\right)$ and consider the sets

$V_{\alpha}^{k}=\left\{x \mid x \in M, \phi_{\mu}(x)<\operatorname{Min}\left[\phi_{\mu_{0}}(x), \cdots, \phi_{\mu_{k}}(x)\right] \mu \neq \mu_{0}, \cdots, \mu_{k}\right\}$.

Each $V_{\alpha}^{k}$ is open and $V_{\alpha}^{k} \cap V_{\beta}^{k}=\varnothing$ for $\alpha \neq \beta$. Furthermore,

$$
V_{\alpha}^{k} \subset\left(\text { carrier } \phi_{\mu_{0}}\right) \cap \cdots \cap\left(\operatorname{carrier} \phi_{\mu_{k}}\right) .
$$


Hence $\bar{V}_{\alpha}^{\boldsymbol{k}}$ is compact and contained in some $W_{\mu}$. Therefore it is contained in a coordinate neighborhood. Since the order of the covering $\left\{W_{\mu}\right\}$ is $n_{0}$, for $k>n_{0}$ the sets $V_{\alpha}^{k}$ are void. The sets $V_{\alpha}^{\mathbf{k}}\left(0 \leqq k \leqq n_{0}\right)$ cover $M$ since for every $x \in M$ some $\phi_{\mu}(x)>0$. The covering $\left\{V_{\alpha}^{k}\right\}$ is locally finite since $\left\{W_{\mu}\right\}$ is and hence it has all desired properties.

THEOREM. The homomorphism $h$ is an isomorphism onto $T_{p+q}$.

Proof. Consider the covering

$$
M=\bigcup_{k=0}^{n_{0}} \bigcup_{\alpha} V_{\alpha}^{k}
$$

constructed in Lemma IV. Since $M$ is paracompact and the covering $\left\{V_{\alpha}^{k}\right\}$ is locally finite we can choose an open subset $W_{\alpha}^{\boldsymbol{k}}$ in each $V_{\alpha}^{\boldsymbol{k}}$ such that

$$
\bar{W}_{\alpha}^{k} \subset V_{\alpha}^{k}
$$

and

$$
\bigcup_{k=0}^{n_{0}} \bigcup \begin{aligned}
& k \\
& W_{\alpha}
\end{aligned}=M
$$

It follows from (7) and the property (i) in Lemma IV that the closures $\bar{W}_{\boldsymbol{\alpha}}^{\boldsymbol{k}}$ are compact.

Put

$$
W^{k}=\bigcup_{\alpha} W_{\alpha}^{k} \quad(k=0 \cdots n)
$$

and let $f^{k}$ be a partition of unity subordinate to the covering $\left\{W^{k}\right\}$. Given an arbitrary tensor field $\Omega \in T_{p+q}$ consider the tensor fields $\Omega^{k}=f^{k} \Omega$. The carrier of $\Omega^{k}$ is contained in $W^{k}$. Applying Lemma II with

$$
U_{\alpha}=V_{\alpha}^{k} \text { and } A_{\alpha}=\bar{W}_{\alpha}^{k},
$$

we see that $\Omega^{k}$ can be written as

$$
\Omega^{k}=\sum_{(\nu)} \lambda_{\nu_{1}}^{k} \cdots \nu_{p+q} \omega^{\nu_{1}} \cdots \omega^{\nu^{p+q}}
$$

where $\omega^{\nu} \in T_{1}$ and $\lambda_{\nu_{1}}^{\boldsymbol{k}} \ldots \nu_{p+q} \in F$. Introducing the tensor fields

$$
\Phi^{\nu_{1} \cdots \nu_{p}}=\omega^{\nu_{1}} \cdots \omega^{v_{p}}
$$




$$
\Psi^{\nu_{p+1}} \cdots \nu_{p+q}=\omega^{\nu_{p+1}} \cdots \omega^{\nu p+q}
$$

we obtain from (9)

$$
\begin{aligned}
\Omega^{k} & =\sum_{(\nu)} \lambda_{\nu_{1} \cdots_{\nu_{p+q}} \Phi^{\nu_{1}} \cdots \nu_{p}}^{k} \Psi^{\nu_{p+1}} \cdots \nu_{p+q} \\
& =h\left(\sum_{(\nu)} \lambda_{\nu_{1} \cdots \nu_{p+q}}^{k} \Phi^{\nu_{1}} \cdots \nu_{p} \otimes \Psi^{\nu_{p+1}} \cdots \nu_{p+q}\right) .
\end{aligned}
$$

Summation over $K$ yields

$$
\Omega=h\left(\sum_{k=0}^{n} \sum_{(\nu)} \lambda_{\nu_{1} \cdots \nu_{p+q}}^{k} \Phi^{\nu_{1} \cdots \nu_{p}} \otimes \Psi^{\nu_{p+1} \cdots \nu_{p+q}}\right) .
$$

This relation shows that $h$ is an onto map.

To prove that $h$ is one-to-one suppose that

$$
h\left(\sum_{j} \Phi^{j} \otimes \Psi^{j}\right)=0
$$

where

$$
\Phi^{j} \in T_{p} \text { and } \quad \Psi^{j} \in T_{q} .
$$

Then $\sum_{j} \Phi \cdot j \Psi^{j}=0$ and multiplication by $f^{k}$ yields

$$
\sum_{j} f^{k} \Phi^{j} \Psi^{j}=0 .
$$

Since the carrier of $f^{k} \Phi^{j}$ is contained in $W^{k} \subset U_{\alpha} \bar{W}_{\alpha}^{k}$ we can apply Lemma III with

$$
U_{\alpha}=V_{\alpha}^{k} \text { and } A_{\alpha}=\bar{W}_{\alpha}^{k} .
$$

We thus obtain

$$
\sum_{j} f^{k} \Phi^{j} \otimes \Psi^{j}=0
$$

and summing over $k$

$$
\sum_{j} \Phi^{j} \otimes \Psi^{j}=0 .
$$

The above theorem is thereby proved.

UNIVERSITY OF TORONTO 